\title{
Dynamics of Plasma Blobs in a Shear Flow
}

\author{
A. Diallo, ${ }^{*}$ A. Fasoli, I. Furno, B. Labit, M. Podestà, ${ }^{+}$and C. Theiler \\ Centre de Recherches en Physique des Plasmas Association Euratom-Confédération Suisse, \\ Ecole Polytechnique Fédérale de Lausanne (EPFL), Lausanne CH-1015, Switzerland \\ (Received 5 March 2008; published 11 September 2008)
}

\begin{abstract}
The global dynamic of plasma blobs in a shear flow is investigated in a simple magnetized torus using the spatial Fourier harmonics (k-space) framework. Direct experimental evidence of a linear drift in $\mathbf{k}$ space of the density fluctuation energy synchronized with blob events is presented. During this drift, an increase of the fluctuation energy and a production of the kinetic energy associated with blobs are observed. The energy source of the blob is analyzed using an advection-dissipation-type equation that includes blob-flow exchange energy, linear drift in $\mathbf{k}$ space, nonlinear processes, and viscous dissipations. We show that blobs tap their energy from the dominant $\mathbf{E} \times \mathbf{B}$ vertical background flow during the linear drift stage. The exchange of energy is unidirectional as there is no evidence that blobs return energy to the flow.
\end{abstract}

In magnetic fusion devices, it is well known that substantial amounts of plasma appearing as coherent structures elongated [1] along the magnetic field lines can propagate to the outer wall, ultimately causing erosion problems. A fair amount is already known about the propagation of these coherent structures; relatively little is understood about their generation and their interaction with the background sheared flow. The velocity shear in a flow is a source of diverse energy-intensive processes (e.g., coherent structures, vortices), which occur in astrophysical objects, in hydrodynamics, in laboratory plasmas, and biological systems. Vortex and coherent structure dynamics are being actively investigated in order to address the recurring dilemma caused by the relationship between the onset and the maintenance of turbulence in shear flows [2].

Recent investigations in plasma physics have revealed the presence of coherent structures of density (also referred to as blobs) in the scrape-off layer of tokamaks [1,3], in simple magnetized torus (SMT) [4,5], and in linear devices [6,7]. In addition to the presence of blobs in SMT, the existence of a shear in the background $\mathbf{E} \times \mathbf{B}$ flow velocity was recently shown in Ref. [5]. In this Letter, we focus on the energy exchange dynamics of blobs with the background flow in the presence of shear. In particular, we present a global analysis in $\mathbf{k}$ space of plasma fluctuations in the presence of intermittent and coherent structures also known as plasma blobs in TORPEX [8] with no prior Fourier decomposition in time. This approach, also referred to as non-normal, is generally utilized to better capture the time evolution of these structures in $\mathbf{k}$ space. First, within this framework, we show experimental evidence of a linear drift of radial wave numbers in time. This drift is associated with an increase of the kinetic energy of the fluctuations associated with the blob, and is similar to numerical investigations of self-sustained coherent vorti- ces in a shear flow reported by Kim and co-workers [9]. In addition to the drift, we deduce the blob time of flight to the edge of the plasma cross section. Second, the key question pertaining to the source of energy provided to the blobs is addressed using an advection-dissipation-type equation that includes blob-flow exchange energy, linear drift in $\mathbf{k}$ space, nonlinear processes, and viscous dissipations.

The experiments are performed on a magnetized plasma contained in the toroidal device TORPEX. The major radius is $R=1 \mathrm{~m}$; the minor radius is $a=20 \mathrm{~cm}$. In the present experiment, hydrogen plasmas are obtained under a regulated neutral pressure of $3.5 \times 10^{-5}$ mbar with a microwave power of $400 \mathrm{~W}$, a toroidal magnetic field of $76 \mathrm{mT}$ on axis on which a vertical magnetic field of $2.3 \mathrm{mT}$ is superposed. Under these field parameters, we found that the lifetime of plasma blobs is sufficiently long to improve their detection and tractability using arrays of Langmuir probes. These probes are used to obtain plasma parameters such as density, temperature, and potential. The electron and ion temperatures are $\sim 4 \mathrm{eV}$ and $\leq 0.1 \mathrm{eV}$, respectively, for an electron density of the order of $10^{16} \mathrm{~m}^{-3}$.

The temporal evolution of the two-dimensional profiles of plasma parameters can be readily reconstructed using the conditional sampling technique (hereafter CS) defined as $\langle X(\tau, r, z)\rangle_{\mathrm{CS}} \doteq\left\{X(t, r, z) \mid Y\left(t, r_{0}, z_{0}\right) \geq \sigma\right\}$, where $X(t, r, z)$ represents the spatiotemporal evolution of a plasma parameter, $\tau$ is the time window centered around the event matching an imposed condition, which in our case is a blob event. $Y\left(t, r_{0}, z_{0}\right)$ is the reference probe signal (located at $r_{0}=14 \mathrm{~cm}$ and $z_{0}=0$ ), and $\sigma$ is the threshold condition imposed (typically expressed in terms of the reference signal standard deviation). First utilized in hydrodynamics to examine the existence of conditional flow structures [10], this technique has since been generalized by Johnsen and co-workers [11] in plasma turbulence and is being used to synchronize signals (e.g., ion saturation 
current, potential) obtained from the plasma with conditional events. The obtained conditional signals enable the reconstruction of the spatiotemporal behavior of fluctuating quantities over a plasma cross section (see Ref. [11] for a description of this technique).

An implementation of CS with an additional trigger condition is performed and is given by $\langle X\rangle_{\mathrm{CS}}=\{X(t, r, z) \mid(1+$ $\left.j / 2) \sigma \leq Y\left(t, r_{0}, z_{0}\right) \leq(1.2+j / 2) \sigma\right\}$, where $j \in\{0, \ldots, 7\}$. This implementation carefully selects an amplitude band of the detected signals (see Refs. [5,12] for a detailed description), which is equivalent to the height of blobs; for brevity, this amplitude band is later referred to as "blob height." Hence, fluctuations in density, in plasma potential, and in electron temperature can be resolved in space (e.g., over a $24 \mathrm{~cm} \times 22 \mathrm{~cm}$ spatial grid) and in time for different blob heights. The 2D fluctuating plasma parameters are reconstructed with a spatial resolution of 1.8 and $1 \mathrm{~cm}$ on the vertical and radial directions, respectively. Such reconstruction is necessary to undertake the Fourier analysis in space.

To better capture novel dynamical features inherent to fluctuations associated with blobs given the enhanced spatial reconstruction, we find it convenient to represent the CS data in $\mathbf{k}$ space. We follow the standard decomposition procedure: $\quad X(r, z, \tau, \sigma)=\int d \mathbf{k} X_{\mathbf{k}}(\mathbf{k}, \tau, \sigma) \exp \left(i k_{r} r+\right.$ $i k_{z} z$ ), where $X$ defines the conditionally averaged plasma parameter, and $\sigma$ represents blob height. This decomposition is also referred to as spatial Fourier harmonics (SFH) (see Chagelishvili et al. $[13,14]$ and references therein). We investigate the evolution of the SFH of fluctuations without any prior spectral decomposition in time in order to capture transients related to blob dynamics.

Figure 1 shows an example of the SFH decomposition of the density fluctuation energy associated with the blobs. Each panel (a) through (h) of Fig. 1 represents the fluctuation energy (symmetric in $|\mathbf{k}|$ ) at two different time frames for distinct blob heights. The panels clearly show a tilting in $\mathbf{k}$ space as a function of time and blob height. This tilting process is further emphasized in Figs. 1(i)-1(k) for a specific blob height $(\sigma=4.5)$, and suggests that the fluctuation energy initially at $k_{r} \sim 0$ is advected toward larger values of radial wave number. The total density fluctuations averaged over all possible $\mathbf{k}$ sustained by the system for each represented $\sigma$ are shown in Fig. 1(1). The vertical dotted line is the corresponding time associated with the panels above. At a given time relative to the detection of blob event (time $=0$ ), different tilting patterns are observed in Figs. 1(a)-1(d) or in Figs. 1(e)-1(h): this suggests a blob-height-dependent tilting process.

Figure 2(a) represents the time evolution of the radial wave number, obtained using a 2D fit (in $k_{r}$ and $k_{z}$ ) of the decomposed density fluctuations for each time frame (note $k_{z}$ is constant in time). This figure shows the first reported experimental evidence of the linear "drift" of the radial wave number towards larger values following the ansatz $k_{r}^{\text {eff }}(\tau)=k_{c}+A k_{z}\left(\tau-\tau_{c}\right) ; A$ is positive and characterizes

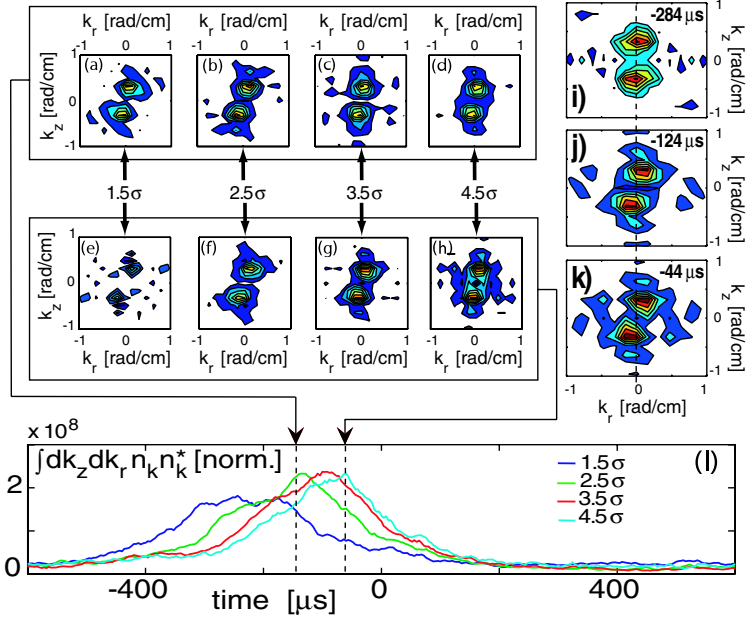

FIG. 1 (color online). Decomposition in $\mathbf{k}$ space of the density fluctuations for two distinct time frames for different blob heights (a) $1.5 \sigma$, (b) $2.5 \sigma$, (c) $3.5 \sigma$, (d) $4.5 \sigma$. (e)-(h) Same as (a)-(d) for a different time. Note that the two peaks are complex conjugate of each other. (f) Fluctuation energy integrated over k for corresponding blob heights: the dashed lines represent the time at which the density fluctuations energy panels are shown. (i)-(k) Example: Illustration of the tilting process (the dashed line represents the reference $k_{r}=0$ ).

the shear of the velocity field, and $k_{z}$ is the vertical wave number of the primary instability. This is termed as the enhanced shear dispersion $[2,15]$ to describe the continuous stretching of the fluctuation structure in the streamwise direction (i.e., in the vertical direction $\hat{e}_{z}$ imposed by the dominant $\mathbf{E} \times \mathbf{B}$ flow). We observe (see Fig. 2 dashed line) that the energy peaks at $\tau_{c}$ corresponding to $k_{c}$ (note that no evidence of $\sigma$ dependence in the shear parameter $A$ is found). The best linear fit of Fig. 2(a) over the most significant part (i.e., the region where the normalized fluctuation energy is greater than $50 \%$ as illustrated in

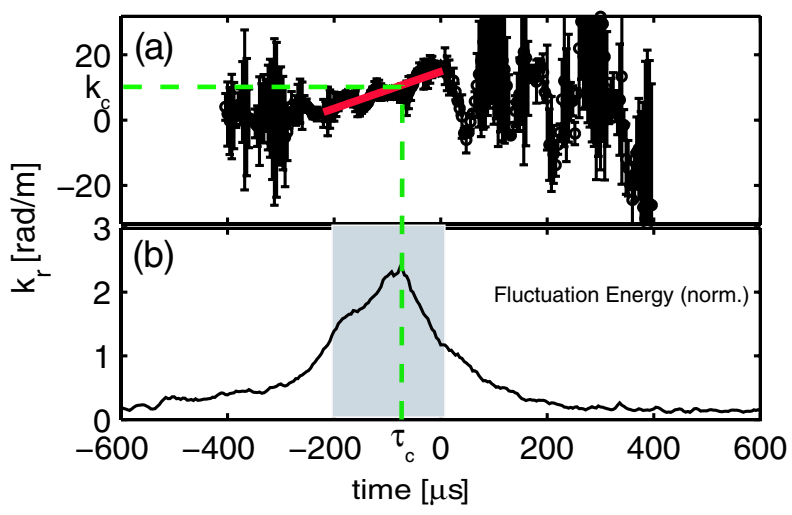

FIG. 2 (color online). Evidence of the linear "drift." (a) The time evolution of $k_{r}$ showing the linear variation of the radial wave numbers illustrated by a solid red line. (b) The blobassociated density fluctuation energy. Note that the shaded area illustrates the corresponding region over which the linear drift is observed. The energy peaks at $\tau_{c}$ corresponding to $k_{c}$ as shown by the dashed green line. 
Fig. 2(b) by the shaded area) yields a shear rate of $3 \mathrm{kHz}$ with $k_{z} \sim 30 \mathrm{rad} \mathrm{m}^{-1}$ being the vertical wave number of the primary instability [see Figs. 1(a)-1(h)]. Note that the primary instability is of the drift-interchange type, and has been extensively discussed in Refs. [5,16].

A time delay in the $\mathbf{k}$-space tilting is clearly observed for blobs of different heights. This delay corresponds to the delay between the time at which the energy peaks (i.e., blob characteristic time $\tau_{c}$ ) and the time at which the blob is detected by the reference probe (at $r_{0}=14 \mathrm{~cm}$ and $\tau=$ $0)$. Generally, the time when the energy peaks corresponds to the birth of a blob. Hence, the delay time applies solely to the already born blob. Figure 3 shows the linear dependence between the measured inverse delay time (also referred to as time of flight) of the fluctuation energy associated with the blob and the square root of its detected height. Note that the difference in detected heights of blobs reflects similar initial differences of blob heights at birth assuming a constant rate $\left(2 c_{s} / L_{\|}\right)$of particle losses along the field lines. Hence, the detected large (small) heights are generated by initially big (small) blobs at birth, from which it follows that big blobs travel faster than smaller ones.

The SFH decomposition of the 2D density fluctuation energy associated with the blob event revealed a linear drift of the radial wave number that is correlated with an increase of the $\mathbf{k}$-averaged fluctuation energy as shown in Fig. 2(b). Figure 4 represents the time evolution of both the total density fluctuations and the computed kinetic energy for each blob height, further investigating the processes occurring during the linear drift. The contour plot in Fig. 4 (a) represents the time evolution of the computed kinetic energy $\left(\int d k_{r} d k_{z}[\nabla \tilde{\varphi} / B]^{2}\right)$ for blob-associated fluctuations of distinct height. Figure 4(b) clearly highlights the production of the kinetic energy during the linear drift (shown in the shaded area). During this drift, two processes occur, the blob-associated density fluctuation energy increases [Figs. 4(c) and 4(d)] and kinetic energy is produced. This suggests an energy exchange mechanism between the fluctuations associated with the blobs and the background $\mathbf{E} \times \mathbf{B}$ flow.

Generally speaking there exist different types of perturbed energies (i.e., kinetic, compressional, and magnetic)

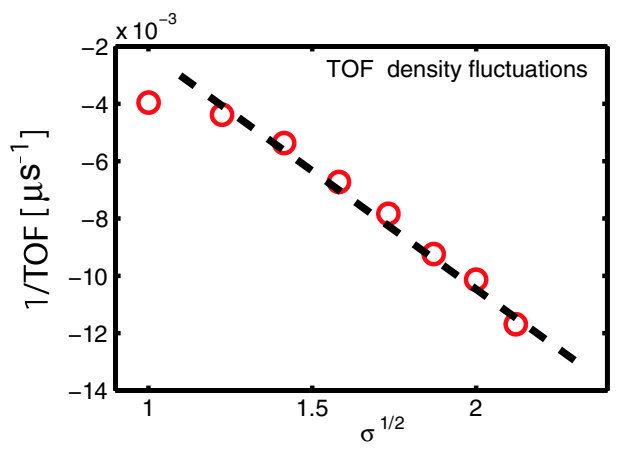

FIG. 3 (color online). The inverse delay time (inverse time of flight) of the blob associated density fluctuations varies linearly with the square root of blob heights. in a system, whose dynamical equations can be considered independently. However, exploring blob-associated density perturbations with dominating kinematics, we use an advection-type dynamical equation, which includes the advection of radial wave number toward larger values, a blob-flow exchange energy, and viscous dissipation and nonlinear processes, to describe the time evolution of the blob-associated spectral energy. Note that this equation is the weak turbulence equation given in the Appendix of Ref. [17] as follows:

$$
\frac{\partial E_{\mathbf{k}}}{\partial \tau}+\nabla_{\mathbf{k}}\left(\mathbf{V} E_{\mathbf{k}}\right)-\frac{2 k_{r} k_{z}}{\mathbf{k}^{2}} A E_{\mathbf{k}}+\nu \mathbf{k}^{2} E_{\mathbf{k}}=N E_{\mathbf{k}} .
$$

Here, $\mathbf{V}$ is the streamwise advection term and is defined as $A k_{r} \hat{e}_{z}$, and $E_{\mathbf{k}}=|\tilde{n}(\mathbf{k}, \tau)| /\left[k_{z}^{2} \mathbf{k}^{2}\right]$ represents the blobassociated perturbation energy density. The term $N E_{\mathbf{k}}$ is the nonlinear term representing the exchange energy between spectral modes. In Ref. [17], it is clearly shown that this term vanishes as it is only responsible for redistributing energy between different SFHs, and therefore does not cause a variation of the total energy. Likewise, the term $\int d \mathbf{k} \nabla_{\mathbf{k}}\left(\mathbf{V} E_{\mathbf{k}}\right)$ vanishes. The third term represents the exchange energy between the blob-associated perturbation and the background vertical flow. The fourth term describes the dissipation that converts the residual energy density into heat through the viscosity $\nu$.

Figure 5(a) represents the time rate of change of the total energy associated with the blob (left vertical axis) and the power associated with the exchange term (right axis). Integrating Eq. (1) over both $\mathbf{k}$ and $\tau$, one obtains in Fig. 5(b) the time evolution of the blob energy (dotted line) and the exchange energy (solid line) on the same energy and time axis. This figure suggests that the blob energy peaks during the linear drift phase. During this same phase (shaded area), an increase of the exchange term (solid line) is observed, which suggests that energy is drawn from the shear flow. Both energies are comparable
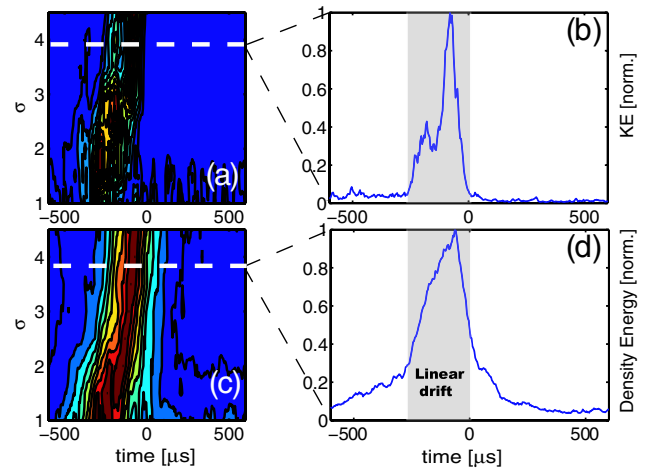

FIG. 4 (color online). (a) Contour plots of the time evolution of the blob-associated kinetic energy as a function of blob heights. (b) Time evolution of the kinetic energy for $\sigma=4$. (c) Similarly, contour plots of the time evolution of the blobassociated density fluctuations energy. (d) Density fluctuation energy for $\sigma=4$. The shaded area represents the linear drift region. 

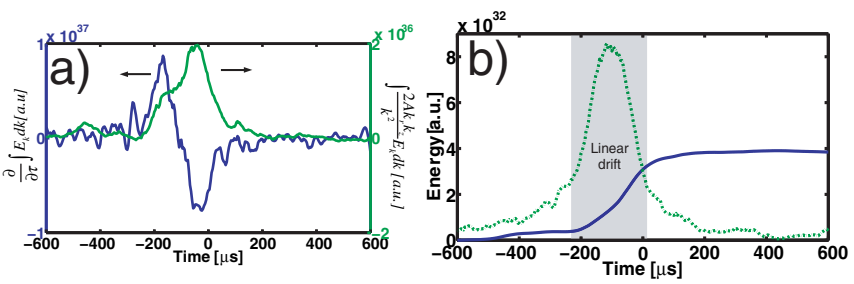

FIG. 5 (color online). (a) (Left vertical axis) The time rate of change of the k-averaged energy associated with the blob of height $\sigma=4\left(\partial_{\tau} \int E_{k} d k\right)$. (Right vertical axis) The power associated with the energy exchange term $\left(\int 2 A k_{r} k_{z} E_{k} / k^{2} d k\right)$. (b) Integrating Eq. (1) over time yields the energy associated with the blob (dotted line) and the energy exchange with flow (solid line) on the same vertical and horizontal axis.

in magnitude thereby implying that blobs tap their energy during the linear drift stage through the shear flow. In other words, the background flow provides energy to the blobs. This exchange, however, is unidirectional as the energy exchange term saturates outside the linear drift region (shaded area). Hence, the energy acquired by blobs is not pumped back into the background flow. This is somewhat apparent as the factor $A k_{r}$ remains positive. Instead, another mechanism (e.g., viscous dissipation) of conversion of the residual energy switches on as $k_{r}(\tau)$ reaches a critical value. This critical value corresponds to the radial wave number where the exchange term becomes larger than the energy associated with blob disturbance. In TORPEX plasmas, such dissipation can presumably occur along the magnetic field lines.

To summarize, we have analyzed the blob dynamics in $\mathbf{k}$ space in the presence of a shear flow. This global analysis involves a spatial Fourier harmonics decomposition over a spatial grid [24 cm $\times 22 \mathrm{~cm}$ ] of time-resolved fluctuation measurements of the main plasma parameters (i.e., density, potential). The decomposition shows that the inverse time of flight of blobs varies with the square root of their heights, implying that larger blob heights propagate faster than smaller ones. In addition, this decomposition reveals a linear drift of the radial wave number with a constant shear parameter (as the primary instability has a constant vertical wave number). This drift is seen as an advection of the radial wave number towards larger values, during which two processes occur: the blob-associated energy increases and kinetic energy is produced. These processes are analogous to the dynamics of vesicles in an external flow [18], where the thermal noise intensification implies an acceleration of the tumbling. Here, the increase of the blobassociated energy is related to the generation of flows through an exchange mechanism.

The exchange mechanism of blob energy is analyzed using an advection-dissipation-type equation that conveniently describes the temporal evolution of the fluctuation energy associated with the blob disturbance. Balancing the different terms of the equation enables us to account for the source of the energy supplied to the blob for it to exhibit a transient growth. As the fluctuations are stretched (during linear drift stage) by the sheared vertical flow, kinetic energy associated with the blob is produced. This production process results from the exchange of energy from the $\mathbf{E} \times \mathbf{B}$ vertical flow to the blob, and therefore analogous to the production of positive Reynolds stress. Comparing the fluctuation energy associated with blob disturbance and the energy exchange term, we find that the two types of energy are of the same order in magnitude during the linear drift stage. Therefore, energy is supplied to blobs through the shear flow. The reverse flow of energy, however, is not necessarily true since the energy exchange term saturates. Hence, energy gained by the blob is not pumped back into the flow and the exchange is unidirectional.

A. D. gratefully acknowledges George Chagelishvili and Ben McMillan for useful discussions. This work was supported in part by the Fonds National Suisse de la Recherche Scientifique.

*ahmed.diallo@anu.edu.au

Present address: Plasma Research Lab, RSPhysSE, Australian National University, Canberra, ACT 0200, Australia.

${ }^{+}$Present address: University of California, Irvine, CA 92697, USA.

[1] S. Krasheninnikov, Phys. Lett. A 283, 368 (2001).

[2] P. A. Davidson, Turbulence (Oxford University, New York, 2005).

[3] J. A. Boedo et al., Phys. Plasmas 10, 1670 (2003).

[4] S. H. Muller et al., Phys. Plasmas 14, 110704 (2007).

[5] I. Furno et al., Phys. Rev. Lett. 100, 055004 (2008).

[6] T. A. Carter, Phys. Plasmas 13, 010701 (2006).

[7] T. Windisch, O. Grulke, and T. Klinger, Phys. Plasmas 13, 122303 (2006).

[8] A. Fasoli et al., Phys. Plasmas 13, 055902 (2006).

[9] J.-H. Kim et al., Phys. Plasmas 13, 062304 (2006).

[10] R. J. Adrian, Phys. Fluids 22, 2065 (1979).

[11] H. Johnsen, H. L. Pecseli, and J. Trulsen, Phys. Fluids 30, 2239 (1987).

[12] M. Podesta, Ph.D. thesis, Ecole Polytechnique Federale de Lausanne, Switzerland, 2007.

[13] G. D. Chagelishvili et al., Phys. Rev. Lett. 79, 3178 (1997).

[14] G. D. Chagelishvili, R. Chanishvili, and D. G. Lominadze, JETP Lett. 63, 543 (1996).

[15] O.E. Garcia and N.H. Bian, Phys. Plasmas 12, 014503 (2005).

[16] F. M. Poli et al., Phys. Plasmas 13, 102104 (2006).

[17] G. Chagelishvili, T.H. R. Chanishvili, and J. Lominadze, JETP 94, 434 (2002).

[18] V. V. Lebedev, K.S. Turitsyn, and S.S. Vergeles, Phys. Rev. Lett. 99, 218101 (2007). 